\title{
Influence of the radiation diffraction in image converter of the thermograph upon its metrological parameters
}

\author{
by S. Poloszyk ${ }^{1}$ and L. Rózañski1. \\ 1 Poznan University of Technology Institute of Mechanical Technology, Division of Metrology and \\ Measurement Systems, Pl. M. Sklodowskiej-Cunie 5, 60-965 Poznañ, Poland
}

\begin{abstract}
The influence of radiation diffraction upon geometrical resolution of the thermograph with series structure of measuring line has been analysed in the paper. Analysis of thermograph properties in bands of $3-5 \mu \mathrm{m}$ and $8-10 \mu \mathrm{m}$ proved that when applying the aperture diaphragms the influence of the radiation diffraction upon geometrical resolution may by significant. This influence is stronger for larger values of the hole numbers in the applied diaphragms. It was shown that the thermal resolution of the thermography system can be improved by reducing the bandnwidth of the electronic system of the camera.
\end{abstract}

\section{Introduction}

The correctness of thermal transformations obtained by means of thermographic equipment is a function of several factors which are dependent or independent on the equipment construction. The dependence on construction factors that are of significant influence upon the quality of thermal transformations, are parameters of the transformation synthesis system. These construction factors/parameters of elements used in the thermograph measuring line determine values of the metrological parameters of the equipment. The most important of them are: thermal resolution (TR) $\Delta T$ and geometrical resolution (GR) $\Delta G$. Construction parameters of the image analysis system that influence the TR and GR are different for different measuring conditions (e.g. filters and diaphragms change parameters of the optical line ) [1].

The influence of radiation diffraction upon the thermograph GR and (what is connected with $\Delta$ T) upon the frequency matching of the image analysis system to representation synthesis system for diaphragms with large hole numbers $N$, has been analysed in the paper ( $N=f / D$, where: $f$ equivalent focal length of the optics, D - diameter of entrance aperture of the optics). All the investigations have been performed for thermographs with series structure of measuring line with rectangular trajectory of image analysis.

\section{Thermal and geometrical resolution of thermograph}

\subsection{Thermal resolution}

Thermal resolution is defined as the capability to distinguish between two temperatures which are very close to each other. There are several methods, known in the literature, to define this parameter. In this paper $\Delta T$ is defined as the temperature difference equivalent to the noise (NETD ). So, $\Delta T$ can be described as [2]:

$$
\Delta T=\frac{2 \sqrt{2 \pi} N^{2} \sqrt{f_{g}}}{a \int_{\lambda_{1}}^{\lambda_{2}} \tau_{0 \lambda} D^{*}{ }_{\lambda} \frac{\partial m_{\lambda}}{\partial T} d \lambda}
$$

where: $\quad f g$ - boundary frequency of the thermograph electronic line,

a - the side of the square effective surface of the detector,

$\tau_{a l}$ - the spectral coefficient of transmissivity of the optical system,

$D_{\lambda}^{*}$ - spectrum detectivity of detector,

$\mathrm{T}$ - absolute temperature,

$m_{\lambda}$ - emittance. 


\subsection{Geometrical resolution}

The geometrical resolution $\Delta G$ is defined as the thermograph ability to reproduce the geometrical details in the thermal image. There are many ways in literature to define this parameter. It seems that the best way to describe the geometrical details is the description of these abilities by the modulation transfer function (MTF). The MTF function is the dependence on spatial frequency, the modulus of the standard Fourier transform out of the distribution function of lines describing the response of the thermograph measuring line against the radiator in the shape of an infinite thin line (located perpendicularly to the scanning direction) where the spatial continuity is preserved in the thermal transformation [3]. So the geometrical resolution $\Delta G$ is such a value of spatial frequency defined in the image plane where the $M T F_{n}=0.5$.

$$
\begin{aligned}
& \Delta G=f_{p x g} \\
& M T F_{n}\left(f_{p x g}\right)=0,5
\end{aligned}
$$

where: $f_{p x g}$ - linear spatial frequency determined in the image plane for $M T F_{n}=0.5$.

\section{Factors determining the modulation transfer function}

The independent members of the thermograph measuring line are: optical system (MTF no), detector $\left(M T F_{n d}\right)$, coupling of the detector with the electronic system with preamplifier $\left(M T F_{n p}\right)$, vision amplifier $\left(M T F_{n w}\right)$, indicator $\left(M T F_{n i}\right)$.

The MTF $n$ function of the thermograph may be described as the product of the particular MTF functions of the members in this measuring line:

$$
M T F_{n}=M T F_{n o} M T F_{n d} M T F_{n p} M T F_{n w} M T F_{n i}
$$

The modulation transfer function (MTF) of any member of the measuring line is defined as the standard Fourier integral transform of the function describing the response of this member to an impulse function. The filtration of the optical system (described by MTF no) is caused by infra-red radiation diffraction and by the aberrations of the optical system (if it is not sufficiently corrected). The detector filtering abilities (described by MTF ${ }_{n d}$ ) are connected with geometrical dimension of its effective surface and with inertia of this element. The electronic line filtration (described by $M T F_{n e}=M_{T F} * M T F_{n w}$ ) is connected with the definite band width of the transmission. Indicator filtration is connected with the finite ability of any element to reproduce the geometrical details. The influence of MTF ${ }_{n i}$ upon the MTF $n$ of the thermograph with the series structure of the measuring line is not significant and it will not be further considered.

\section{Modulation transfer function of the optical system}

Assuming that the optical system of the thermograph is corrected and it has the entrance pupil in the shape of a circle and that it co-operates with the detector of selective spectral characteristic, it is possible to obtain the MTF no defined by the following equations:

$$
M T F_{n o}=\frac{\mid \int_{-\infty}^{+\infty} e_{w n i}(x) \exp \left(-j 2 \pi x f_{x} d x \mid\right.}{\left|\int_{-\infty}^{+\infty} e_{w n i}(x) d x\right|}
$$




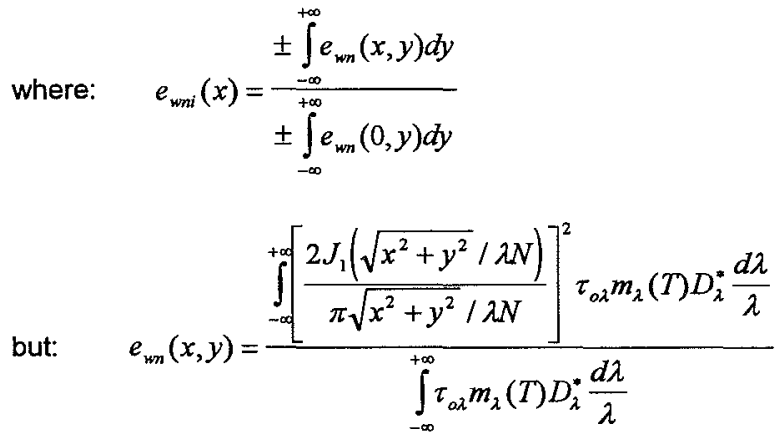

where: $\quad e_{w n}(x, y)$ - standard weighted radiation intensity in the point of $x, y$ coordinates (image plane).

$J_{1}$ - Bessel's function of the first grade.

Equation (6) describes the distribution of standard weighted radiation intensity in the diffraction spot that is the created by the corrected optical system as an image of the point existing in the subject plane. Equations (4) and (6) will be used to determine the influence of filtering abilities of the optical system upon the MTF $n$ of the thermograph.

\section{Modulation transfer function of detector}

It has been assumed that:

- effective surface of the infrared detector used in the thermograph is a regular square,

- one pair of sides of the effective detector is parallel to the direction of line analysis,

- detector is the inertial element of the first grade (only with one time constant).

Based on above assumptions the MTF $_{\text {nod }}$ may by defined as:

$$
M T F_{n d}\left(f_{p x}\right)=\frac{\sin \left(\pi a f_{p x}\right)}{\pi a f_{p x}}\left(1+4 \pi^{2} f_{p x}^{2} v^{2} \tau_{d}^{2}\right)^{-\frac{1}{2}}
$$

where: a - side of the square effective surface of the detector,

$v$ - speed at which the image of an object is moved over the detector surface,

$\tau_{d}$ - time constant of the detector.

Usually a $\geq 0.1 \mathrm{~mm}, \tau_{d} \geq 0.5 \mu \mathrm{s}$ and $v<30 \mathrm{~m} / \mathrm{s}$ so in equation (7) the factor describing the inertia of the detector can be omitted. So

$$
M T F_{n d}\left(f_{p x}\right)=\frac{\sin \left(\pi a f_{p x}\right)}{\pi a f_{p x}}
$$

Equation (8) will be used to determine how the filtering abilities of the detector influence the MTF $_{\mathrm{n}}$ of the thermograph.

\section{Modulation transfer function of the electronic line}

It has been assumed that the band width transmission of the thermograph electronics is limited by the low - pass filter with an attenuation of $20 \mathrm{~dB} / \mathrm{dec}$. Based on this assumption $M T F_{\text {ne }}$ may be determined by: 


$$
\operatorname{MTF}_{n s}\left(f_{p x}\right)=\frac{1}{\sqrt{1+\left(\frac{f_{p x}}{f_{p x g r}}\right)^{2}}}
$$

where:

$$
\begin{aligned}
& f_{p x g r}-\text { boundary spatial frequency of the filter, } \\
& f_{g r}=f_{p x g r} v \\
& f_{g r} \text { - boundary frequency of the filter. }
\end{aligned}
$$

Equation (9) will be used to determine how the characteristics of the electronic filter influences the MTF $\mathrm{n}$.

\section{Influence of diffraction upon MTF}

It has been assumed that the transmission band of the electronic system has been chosen in such a way that there is no influence upon the MTF $n$. So the MTF may determined by the equation:

$$
M T F_{n}=\frac{\sin \left(\pi a f_{p x}\right)}{\pi a f_{p x}} M T F_{n o}
$$

The detectors used in the described thermograph have $a \geq 0,1 \mathrm{~mm}$. Applying equations (4), (5), (6) and (10) it has been stated that when diaphragms are not used, the main factor influencing on MTF $_{n}$ is the spatial filtration of the detector. The influence of the optical system filtration is very small and can be omitted. The course of MTF $n$ has been calculated for $N=1.8, a=0.1 \mathrm{~mm}, \lambda_{1}=$ $3 \mu \mathrm{m}, \lambda_{5}=5 \mu \mathrm{m}, T=300 \mathrm{~K}$ and $\tau_{0 \lambda}=1$ (see curve 2 in figure 2). The MTF $\mathrm{n}$ calculated for a band width from $\lambda_{1}=8 \mu \mathrm{m}$ to $\lambda_{2}=10 \mu \mathrm{m}$ is shown as curve 2 in figure 3 . The situation is different when an optical system is equipped with aperture diaphragms with large hole numbers (this can happen while imaging a high temperature object). The larger the hole numbers in used diaphragms, the stronger is the influence of the optical system filtration upon $M_{T-}$. Curvfes 4 in figures 2 and 3 describe the $M T F_{n}$ for $N=20.4$ and for band widths of $3-5 \mu \mathrm{m}$ and $8-10 \mu \mathrm{m}$, respectively. For particular calculations it has been assumed that $T=1000 \mathrm{~K}$. As it has been shown by curves 2 and 4 , the diffraction of the radiation can spoil the thermograph geometrical resolution by about $31 \%$ in the $3-5 \mu \mathrm{m}$ band and about $60 \%$ in the $8-10 \mu \mathrm{m}$ band. Practically, electronic systems are not realised in such a way that they have no influence on MTF $_{n} . A$ too broad transmission band of the electronic line can spoil $\Delta T$ without any significant influence on $\Delta$ G. Based on literature data it is possible to state that the $f_{p x g r}$ value is determined as:

$$
f_{\mathrm{pxgr}}=\frac{1}{2 a}
$$

When MTF $_{\text {ne }}$ is calculated according to equation (11) its course (urves 1 in figures 2 and 3 ) influences significantly $M T F_{n}$ (curves 3 for $N=1.8$ and curves 5 for $N=20.4$ in figures 2 and 3 ) and at the same time also $\Delta G$ of the thermograph. The diffraction of radiation in this case impairs the geometrical resolution of the thermograph by about $24 \%$ in the band of $3-5 \mu \mathrm{m}$ and about $51 \%$ in the band of $8-10 \mu \mathrm{m}$. The dependence (11) describing the rule of matching the image analysis system with the representation synthesis system connects the value of $f_{p x g r}$ with the dimensions of detector effective surface. It seems that a general rule concerning the way of determining $f_{\text {pxgr }}$ value should connect this value with the modulation transfer function describing spatial filtration of the optical system and the detector. So equation (11) has been replaced by another one where the boundary frequency of the electronic filter is equal to such a spatial line frequency that equation (12) is fulfilled. 


$$
M T F_{n d}\left(f_{p x g r}\right) M T F_{n o}\left(f_{p x g r}\right)=\frac{2}{\pi}
$$

When $M T F_{n o} M T F_{n d}=M T F_{n d}$ the values of $f_{p x g r}$ are equal for equations (11) and (12). Using equation (12) the MTF ne courses have been determined for $N=20.4$ (curves 6 in figures 2 and 3 ). Curves 7 in figures 2 and 3 determine the thermograph $M T F_{n}$ courses with reduced band widths:
1. for $\lambda=3-5 \mu \mathrm{m}$
a/ thermal resolution
b/ geometrical resolution
2. for $\lambda=8-12 \mu \mathrm{m}$
a/ thermal resolution
b/ geometrical resolution
improved by $23 \%$, worse by $11 \%$, improved by $38 \%$, worse by $19 \%$.

The above results are valid only if MTF no is connected only with the diffraction of radiation. The results and conclusions given above are valid only for thermographic systems equipped with aberrationless optical systems. Practically, it is impossible to make optical systems that are aberrationless. Spatial filtration of the real optical systems is connected both with radiation diffraction and with aberration. It depends on the precision with which the optical system has been corrected, manufactured and adjusted, which of the factors will be dominating. Generally, it is possible to state that aberrations of the optical system become smaller with the increase of the hole number $\mathrm{N}$. Physically it can be explained that the strongest aberrations are introduced into the system by boundary zones. It can be stated that application of the aperture diaphragms in optical system with aberrations reduce the aberration spot radius and thereby improve the geometrical resolution of the system. However, in case of well corrected optical systems, the inverse phenomena may occur and impairing of GR is observed because of reaction increase of radiation diffraction (wave nature of radiation makes the increase of diffraction spot diameter with the increase of the hole number $N$ ). As the result the following conclusions can be stated in ependence of diffractional or aberrational factor: GR of thermograph impairs (for small aberrations of the optical system); GR of thermograph improves (for strong aberrations of the optical system); GR of thermograph does not change when the influences of both factors are balanced.

\section{Conclusions}

- Radiation diffraction can significantly influence the GR of the thermograph with the series structure of the measuring line. This influence is stronger for smaller dimensions of the detector effective surface and for larger values of diaphragm hole numbers used during measurements.

- In thermographic systems which are equipped with a sufficiently corrected optical system and with infrared detectors with effective diameters of about $0.1 \mathrm{~mm}$, it is possible, when applying the aperture diaphragms with great hole numbers, to reduce the transmission band of the thermograph electronic line in a way which improves the TR and impairs the GR at the same time.

\section{REFERENCES}

[1] HUDSON (R.) - Infrared System Engineering. J. Wiley and Sons, New York, 1969.

[2] RUDOWSKI (G.) - Termowizja i jej zastosowanie. WKi£, Warszawa, 1979.

[3] VERMEIJ (F.), ELAND (M.), STEKETEE (J). - MTF measurement a simple method for determining the spatial resolution of a thermograph. Acta Thermographica, 1980, No 3. 

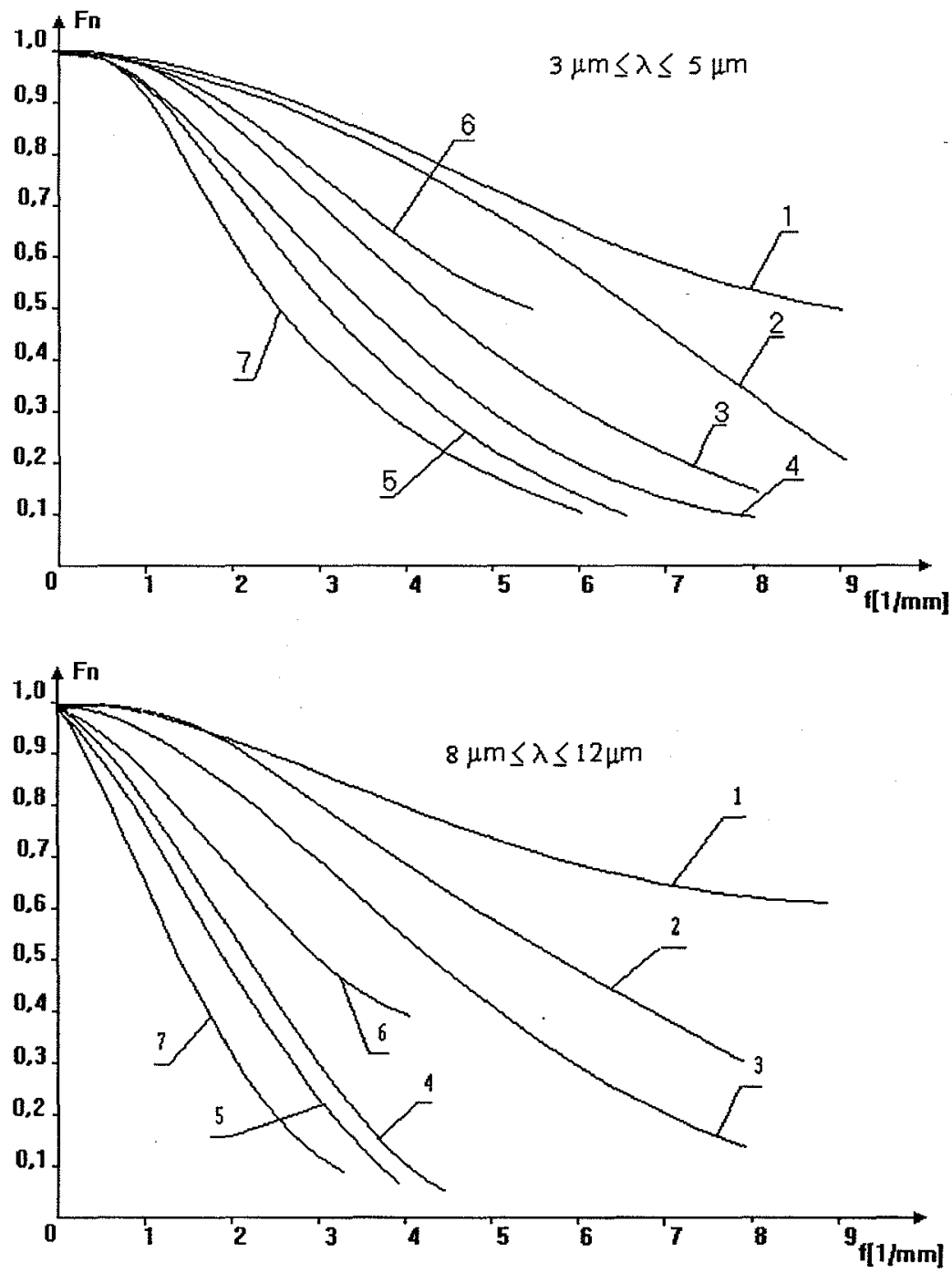

Figure 2 Modulation transfer function of the thermograph measuring line $\left(M T F_{n}\right)$ and modulation transfer function of the thermograph electronic line $\left(M T F_{n e}\right.$ ).

1 - MTF $F_{\text {ne }}$ well-fitting to optical system with $\mathrm{N}=1.8\left(\mathrm{MTF}_{\text {ne1 }}\right)$

$2-M_{T F}$ calculated for $N=1.8, T=300 \mathrm{~K}, a=0.1 \mathrm{~mm}$; it has been assumed that $\mathrm{MTF}_{\text {ne1 }}=1$

$3-M_{n} \quad$ calculated for $N=1.8, T=300 K, a=0.1 \mathrm{~mm} ; M_{T F}$ ne1 $\neq 1$

$4-M_{n}$ calculated for $N=20.4, T=100 K, a=0.1 \mathrm{~mm}$; it has been assumed that $M T F_{n e 1}=1$

$5-M_{n}$ calculated for $N=20.4, T=1000 \mathrm{~K}, a=0.1 \mathrm{~mm}$; it has been assumed that $M T F_{\text {ne }} \neq 1$

$6-M^{M T F}$ well-fitting to optical system with $\mathrm{N}=20.4\left(\mathrm{MTF}_{\mathrm{ne}}\right)$

$7-M_{T} \quad$ calculated for $N=20.4, T=1000 K, a=0.1 m$; $M T F_{n e 2} \neq 1$. 